\title{
Two Years of Computer Supported Outbreak Detection in Sweden: the User's Perspective
}

\author{
Kling $\mathrm{AM}^{*}$, Hebing $\mathrm{K}$, Grünewald $\mathbf{M}$ and Hulth $\mathrm{A}$
}

Swedish Institute for Communicable Disease Control, Solna, Sweden

\begin{abstract}
Computer Assisted Search for Epidemics (CASE) is a framework for computer supported outbreak detection, developed at the Swedish Institute for Communicable Disease Control. The system is aimed to be a complement to the manual surveillance of the notifiable diseases and has been in routine use at the institute since August 2009. In this paper the workflow in the CASE project and the usage from the user's perspective is presented. We describe the CASE team's work, out of the routine document set up for the CASE project. The results of a survey, among the epidemiologists using CASE, are also presented. Our evaluation shows that CASE is a useful and important tool in the routine surveillance work performed by the epidemiologists at the institute. We believe there are several reasons for this success. One is the flexibility of CASE, which allows for different parameter settings for different diseases and the ability to tailor the system as needed. Other reasons are the close collaboration between the CASE team and the epidemiologists and the continuous development of the system which is adapted to the actual needs of the epidemiologists in charge of surveillance.
\end{abstract}

Keywords: Computer-assisted outbreak detection; User evaluation; Infectious disease outbreaks; Public health

\section{Introduction}

Computer Assisted Search for Epidemics (CASE) is a framework for computer supported outbreak detection, developed and in use at the Swedish Institute for Communicable Disease Control (SMI) [1,2]. CASE is aimed to support the epidemiologist in their daily work on the surveillance of the notifiable communicable diseases. The system has been in routine use at SMI since August 2009. The framework is available as open source software [1,2].

CASE enables surveillance of Swedish notifiable diseases using data from the Swedish registry of notifiable diseases (SmiNet) [3]. When the data are extracted from SmiNet by CASE, personal identification codes are removed, and only variables used by CASE are included in the CASE database. Today CASE supports five different statistical algorithms: SaTScan Poisson [4], SaTScan Space-Time Permutation [5], an algorithm developed by Farrington et al. [6], OutbreakP [7] and a simple threshold algorithm. It is possible to extend the system to incorporate additional algorithms. The choice of algorithm(s) and parameter settings can be tailored for the various diseases. The automated analyses are performed daily. If CASE detects a potential outbreak, the signal is sent by an email to the epidemiologist(s) in charge of the surveillance of the pathogen in question.

All 63 notifiable diseases in Sweden are available for computer assisted surveillance with CASE. It is up to each epidemiologist to decide if CASE should be used as a complementary tool for surveillance. Currently, twelve epidemiologists receive automatically generated signals from CASE for the surveillance of around 40 diseases/subtypes. These twelve epidemiologists are responsible for the surveillance of different disease groups: Human Immunodeficiency Virus (HIV), other sexually transmitted diseases and other blood-borne diseases, food- and waterborne diseases and zoonoses, vaccine preventable diseases, and antimicrobial resistance.

In this paper we present the workflow of the daily, practical usage of CASE at SMI. We also present an evaluation of the usefulness of CASE based on a survey conducted among the epidemiologists receiving the signals.

\section{Material and Methods}

\section{Work flow}

The CASE team consists of two statisticians, a system developer and a project leader. The team works closely with the epidemiologists at SMI, discussing possible interpretations of CASE signals and how the system can be tailored to better meet their specific needs. The work in the CASE team emanates from the areas of responsibility and tasks stated in the routine document set up for the CASE project (Table 1).

A schematic illustration of the overall work flow between the system (CASE), project team and epidemiologist are shown in figure 1. When an epidemiologist wants to use CASE for surveillance of a disease $s($ he) is responsible for, (s)he contacts the CASE statistician who arranges a face to face meeting. For a new CASE user the meeting begins with a presentation of CASE, including: the purpose of the system; the graphical user interface (GUI), which is not seen by the end user (the epidemiologist); and how signals are communicated to the end users. Examples of output from the algorithms are given, as is a brief explanation of the different algorithms. This first part is skipped or shortened for an existing CASE user that would like to begin using CASE for a not yet activated disease. The epidemiologist explains the epidemiology of the disease and for what kind of increase in cases (s)he would like to receive CASE signals for to the statistician.

*Corresponding author: Anna-Maria Kling, Swedish Institute for Communicable Disease Control, 17182 Solna, Sweden, Tel: +46 845 72533; Fax: +46 830 0626; E-mail: Anna-maria.kling@smi.se

Received January 03, 2012; Accepted January 24, 2012; Published January 27, 2012

Citation: Kling AM, Hebing K, Grünewald M, Hulth A (2012) Two Years of Computer Supported Outbreak Detection in Sweden: the User's Perspective. J Health Med Informat 3:108. doi:10.4172/2157-7420.1000108

Copyright: (C) 2012 Kling AM, et al. This is an open-access article distributed under the terms of the Creative Commons Attribution License, which permits unrestricted use, distribution, and reproduction in any medium, provided the original author and source are credited. 
Based on the discussions one or more algorithms are chosen, together with values for parameter settings. Occasionally the CASE team will additionally approach the epidemiologists to discuss if and how CASE can be extended to suit more pathogens than the once already under surveillance.

\section{User survey}

The routine document states that a survey aiming to evaluate the usefulness of CASE, should be conducted every second year. The first survey according to this routine was performed in February 2011. All epidemiologists that were using CASE at SMI at that time $(n=9)$ were sent a web-based questionnaire created in the program Artologik [8]. The questionnaire consisted of thirteen questions concerning three topics: usefulness of the system; collaboration with the CASE team; and what further development of the system that should be prioritized. The questions on the usefulness of the system and the CASE team were asked with respect to the varied time period that the users had been using CASE.

\section{Results}

\section{Work flow}

For every diseases/subtypes which gets activated in CASE, a standardized form is filled out by the statistician. In this form responsible epidemiologist, short description of disease, description of what kind of signals that is of interest, algorithm(s) and parameter setting are documented. If some change in algorithm(s) or/and parameter setting is done this is logged in this form together with the

\begin{tabular}{|c|c|c|}
\hline \multicolumn{3}{|l|}{ Area of responsibility } \\
\hline Project leader: & Statisticians: & System developer: \\
\hline $\begin{array}{l}\text { - Invite and chair } \\
\text { scheduled meetings } \\
\text { Budget planning and } \\
\text { follow-up } \\
\text { - Project documentation } \\
\text { - Content in external } \\
\text { project web site } \\
\text { Prioritise the work for } \\
\text { the statisticians and the } \\
\text { system developer }\end{array}$ & $\begin{array}{l}\text { - Operation of the system } \\
\text { - Regular discussions } \\
\text { with the epidemiologists } \\
\text { - Logging of signals } \\
\text { - Discussions on the } \\
\text { outcomes of signals with } \\
\text { the epidemiologists } \\
\text { - Check CASE email } \\
\text { inbox } \\
\text { - Write specifications of } \\
\text { new functions in the } \\
\text { system }\end{array}$ & $\begin{array}{l}\text { - Programming } \\
\text { - Graphical layout of user } \\
\text { interface } \\
\text { - Back up of source code } \\
\text { - Update external project } \\
\text { web site }\end{array}$ \\
\hline
\end{tabular}

Table 1: Areas of responsibility in the CASE team.

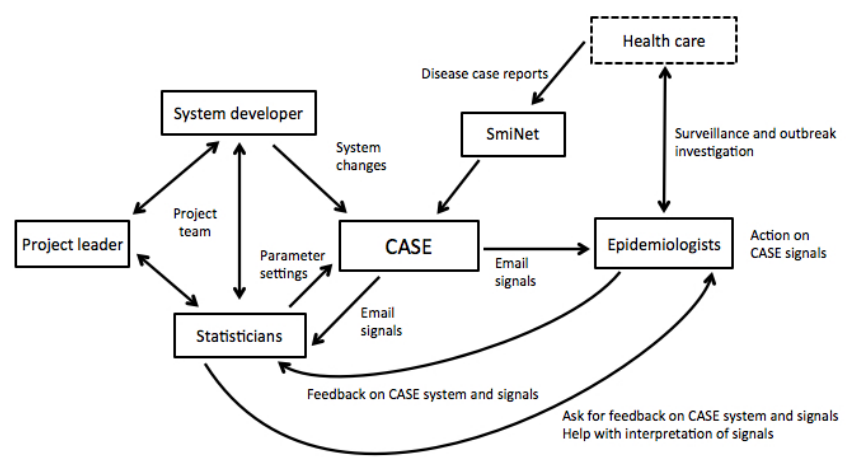

Figure 1: Flow chart of the work processes in CASE, dashed box is located outside SMI. date of change. After the logging procedure, the statistician activates or makes the appropriate changes of the settings through the CASE GUI.

When a new user receives a first signal from CASE, one of the CASE statistician contacts the epidemiologist to further explain and discus the interpretation of the signal. If more than one algorithm is used for the disease, a contact is made every time a new algorithm gives a first signal. During the first time period after activating a new disease in CASE the epidemiologist and the statistician are in close contact to discus if any tailoring of the parameter setting is needed.

All signals from CASE are, in addition to the epidemiologist in charge for the surveillance of the disease, sent to a designated project email address. All signals are screened and archived by a member in the CASE team. For some signals the CASE team also contacts the epidemiologist in charge is for further discussion. When such an action is taken, this is subsequently logged in the disease specific form. The epidemiological follow-up on a signal is, however, always the responsibility of the epidemiologists. The discussion between the statistician and the epidemiologist is for evaluation purposes of the function of the system, and is not required for the epidemiologist to act on the signal.

One of the statisticians is responsible for screening the signals from CASE. During holidays the emails are read according to a planned schedule for the CASE team. This ensures that the emails are always checked with maximum three days delay all days of the year. During season holidays the users are asked if they prefer to alter the recipient of the signals or make a temporary halt. The users are also informed about the CASE team's schedule during the holidays.

The CASE statisticians are the main contact persons for the epidemiologists if they need assistance in interpreting signals, have questions, want to provide feedback in general or have ideas on how the system can be further developed to meet their needs. The close collaboration between the CASE team and CASE users make this contact easy through email, telephone or dropping by the office. All ideas for further tailoring of the system are logged.

\section{User tailoring}

During the time period that CASE has been in regular usage, CASE has been tailored according to the epidemiologist needs, for example:

- The computer based surveillance can optionally be done only for cases where country of infection is Sweden or unknown. That is, cases with a specified country of infection other than Sweden are excluded.

- Information in the output for a generated signal was enhanced with the percentage of cases with unknown country of infection on which the signal is based.

- For Hepatitis B a tailored solution that enables surveillance of only acute cases was implemented.

- A user friendly tool to suppress identical signals in consecutive days was implemented for the algorithm developed by Farrington et al. [6].

Also, the functionality and layout of the GUI have been extended to be more user friendly for the administrator of the system. Examples 
of this are a user manual that is easily accessible in the help menu in the GUI, an easier process of adding or deactivate a user and a function that creates an overview of activated diseases/subtypes and users.

\section{Web based survey}

The survey had a $100 \%$ response rate $(n=9)$. The epidemiologists all agreed that CASE verifies signals seen in their routine surveillance work (Table 2). Eight out of nine thought that the CASE signals give an indication of when cases should be further checked in the SmiNet database of notifiable cases. Seven out of nine stated that CASE saves time in their daily work. Five out of nine stated that they had been made first aware of an outbreak by CASE on at least one occasion. These five epidemiologists are responsible for the surveillance of sexually transmitted infections $(n=3)$ and antibiotic resistant bacteria $(n=2)$. On the other hand, four out of nine did not agree that CASE gives the first signal about an outbreak, three of the respondents thought that CASE gives to many non-relevant signals, and two think that CASE give too many signals overall. Four epidemiologist judged the relevance of the signals to totally relevant/ relevant (Figure 2).

All respondents answered that they know whom to contact when questions about CASE arise and that the questions are answered properly. Eight out of nine thought that their ideas and feedback are received well by the CASE team (one user answered that no such feedback had been given to the CASE team).

\begin{tabular}{|c|c|c|c|}
\hline Question & $\begin{array}{l}\text { Favourable } \\
\text { response }\end{array}$ & $\begin{array}{l}\text { Non favourable } \\
\text { response }\end{array}$ & \\
\hline & Agree & Disagree & Do not know \\
\hline $\begin{array}{l}\text { CASE generates signals for } \\
\text { potential outbreaks before it } \\
\text { can be realized from SmiNet } \\
\text { surveillance. }\end{array}$ & 4 & 4 & 1 \\
\hline \multirow{2}{*}{$\begin{array}{l}\text { CASE signals give an indication } \\
\text { of when cases should be } \\
\text { examined in SmiNet }\end{array}$} & 8 & 0 & 1 \\
\hline & Disagree & Agree & Do not know \\
\hline $\begin{array}{l}\text { CASE fails to give signals for } \\
\text { potential outbreaks }\end{array}$ & 5 & 0 & 4 \\
\hline $\begin{array}{l}\text { CASE gives too many non } \\
\text { relevant signals }\end{array}$ & 6 & 3 & 0 \\
\hline
\end{tabular}

Table 2: Frequency distributions for four questions from the user survey ( $n=9)$.

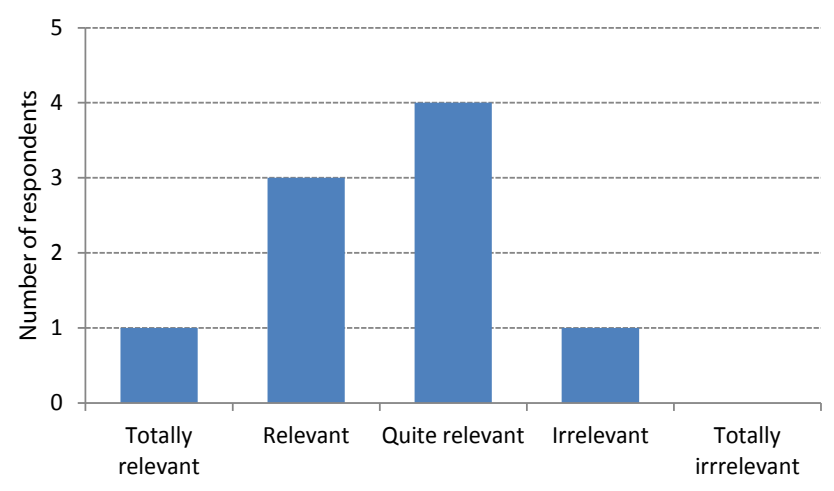

Figure 2: Relevance of CASE signals as judged by the epidemiologists $(n=9)$.
The respondents stated that the most important things to prioritize in the CASE team were to implement a tool to be able to suppress identical signals $(n=5)$, add new algorithms $(n=2)$ and enable for surveillance grouped by different transmission paths $(n=2)$.

\section{Discussion and Conclusions}

The routine document for CASE controls the work in the CASE team and ensures a quality-assured work process. The clear roles for each project member, routines for quality controls and work actions stated in this document makes the work process for CASE smooth. This document is continuously updated as new routines are implemented in the project.

The results from the user survey show that CASE is a valuable and appreciated tool in the daily surveillance work at SMI. Nonetheless and also shown by some results from the survey, there are areas in which CASE can be improved. The main focus is to increase the quality of the signals from CASE. One is to improve the quality of the surveillance data on which CASE bases the signals. This is however something which the CASE team can have little influence over, although this is a continuously ongoing task at the institute. Another way, and an issue that the CASE team can control, is to provide algorithms suitable for the requested surveillance, and allow for informed decision for parameter settings. The survey showed that user wished for a tool to suppress identical signals which was subsequently implemented for the algorithm developed by Farrington et al. [6]. This function will be implemented for the five other supported algorithms during 2012. In the near future, the work with implementing surveillance based on different transmission paths will start with five diseases, namely, HIV, Chlamydia, Gonorrhoea, Syphilis and acute hepatitis B.

In the present situation the CASE team have no plans of adding new algorithms to the system, even though it is a wish from the users. We instead plan to first examine how current algorithms can be improved and tailored for our needs. However, this issue will be addressed again in the future and new algorithm(s) will then be added if it is necessary to improve the quality of the computer assisted outbreak detection.

So far, the quality of CASE signals has been evaluated through communication with the epidemiologists. This is for various reasons not the most optimal way and needs to be improved. In the Swedish database of reported cases of the notifiable diseases, SmiNet, information on whether a case belonged to an outbreak was previously not stored. A new module is currently being added to SmiNet that store this kind of information. In the future this can be an important tool for the CASE team when evaluation the sensitivity and specificity of CASE signals.

Our evaluation shows that CASE is a useful and important tool in the routine surveillance work performed by the epidemiologists at the institute. We believe there are several reasons for this success. One is the flexibility of CASE, which allows for different parameter settings for different diseases and the ability to tailor the system as needed. Other reasons are the close collaboration between the CASE team and the epidemiologists and the continuous development of the system which is adapted to the actual needs of the epidemiologists in charge of surveillance. 
Citation: Kling AM, Hebing K, Grünewald M, Hulth A (2012) Two Years of Computer Supported Outbreak Detection in Sweden: the User's Perspective. J Health Med Informat 3:108. doi:10.4172/2157-7420.1000108

Page 4 of 4

\section{Acknowledgment}

We would like to thank the epidemiologists at SMI for the continuous feedback to the CASE team and their ideas how to improve the system. The project was funded by the Swedish Civil Contingencies Agency.

\section{References}

1. Cakici B, Hebing K, Grünewald M, Saretok P, Hulth A (2010) CASE: a framework for computer supported outbreak detection. BMC Med Inform Decis Mak 10:14

2. CASE: computer assisted search for epidemics [https://smisvn.smi.se/case/].

3. Rolfhamre P, Janson A, Arneborn M, Ekdahl K (2006) SmiNet-2: Description of an internet-based surveillance system for communicable diseases in Sweden. Euro Surveill 11: 103-107.
4. Kulldorff M (1997) A spatial scan statistic. Commun Stat Theory Methods 26: 1481-1496.

5. Kulldorff M, Hartman Heffernan J, Assunção R, Mostashari F (2005) A SpaceTime Permutation Scan Statistic for Disease Outbreak Detection. PLoS Med 2: e59.

6. Farrington CP, Andrews NJ, Beale AD, Catchpole MA (1996) A statistica algorithm for the early detection of outbreaks of infectious disease. J Roy Stat Soc Stat Soc 159: 547-563.

7. Frisén M, Andersson E, Schiöler L (2009) Robust outbreak surveillance of epidemics in Sweden. Stat Med 3: 476-493.

8. Artisan Global Software, Sweden 